\title{
Uncooled detectors for high sensitivity synchronous thermography
}

\author{
Nik Rajic
}

\begin{abstract}
Thermal detectors are seldom considered a viable alternative to cooled photon detectors for scientific thermographic applications. Their inferior noise equivalent temperature difference (NETD) and slower dynamic response are generally assumed to militate against good performance in applications requiring high sensitivity and speed, and for the most part that assessment is true. For the measurement of weak high-speed transients in flash thermography for instance photon detectors are unarguably superior. The same however cannot be said of all synchronous thermographic methods, several of which fulfil important roles in nondestructive inspection and experimental mechanics, e.g. optical lock-in thermography and thermoelastic stress analysis (TSA). For such methods, the signal of interest is not transient but persistent and oscillatory and often so weak as to be undetectable without synchronous averaging. In such cases the ultimate sensitivity achievable by a detector is determined not by its random temporal noise floor for which the NETD is a good figure of merit, but the fixed pattern noise floor a parameter which is seldom specified by manufacturers or measured by the user. This presentation will outline how this distinction explains why microbolometers can achieve performance levels comparable to cooled photon detectors for thermoelastic stress analysis, a finding that has had significant practical implications for the aircraft fatigue community. Case studies are given to illustrate how microbolometer-based TSA applied to airframe full scale fatigue testing has enabled a significant improvement in one of the underpinning elements of aircraft structural certification practice.
\end{abstract}

\section{Biography}

Nik Rajic is Acting Head of the Airframe Diagnostic Systems Group at the Defence Science and Technology Group in Melbourne, Australia. He graduated in 1989 from the University of Melbourne with a Mechanical Engineering degree, and in 1995 from Monash University with a $\mathrm{PhD}$ in Mechanical Engineering. His research interests include non-destructive inspection, structural health monitoring using plate wave acoustics and experimental mechanics. He has over 150 scholarly publications including 5 book chapters and over 40 archival journal papers. He serves on several scientific boards and is an associate editor of the Journal of Structural Health Monitoring. 